\title{
The ATLAS Level-1 Muon Barrel Trigger timing studies using combined trigger and offline tracking
}

\author{
G. Salamanna (NIKHEF), on behalf of the ATLAS Collaboration
}

\begin{abstract}
We present here the strategy and tools developed to time in all the elements of the Level-1 Muon Barrel Trigger of the ATLAS experiment at the CERN Large Hadron Collider. A perfect synchronicity among the various trigger elements and with the LHC Bunch Crossing time is vital for the correctness of the ATLAS physics results. We review the possible sources of delays and how they are accounted and corrected for, using a large sample of cosmic ray data collected by ATLAS; and present initial results, that will be refined with early LHC collision data.
\end{abstract}

\section{INTRODUCTION}

$\mathbf{T}$ HE ATLAS Level-1 Muon Barrel Trigger is one of the main elements of the first stage of event selection of the ATLAS experiment at the Large Hadron Collider. The challenge of the Level-1 system is a reduction of the event rate from a bunch crossing rate of $40 \mathrm{MHz}$ by a factor $10^{3}$, using simple algorithms that can be executed with a latency of the order of $2.5 \mu \mathrm{s}$. The input stage of the Level-1 Muon consists of an array of processors receiving the full granularity of data from dedicated detectors (Resistive Plate Chambers in the Barrel).

Because of the differences in muon time-of-flight, propagation delays on the read-out strips of the RPC and cables and optical fiber lenghts, signals have to be adjusted in time in order to be correctly aligned before being processed. Here we present the analysis technics developed to allow for a study of time misalignements both among the RPC trigger sectors and globally with the LHC clock. These studies collect and integrate information from several sources, such as trigger signal arrival time from the ATLAS Central Trigger Processor, both from the RPC and external triggers (e.g. the Inner Detector); and offline reconstructed muon tracks. These techniques have been tested using a large statistics sample of cosmic muon events in combined runs with the rest of the ATLAS detector; and are expected to provide a fast and detailed mapping of the status of the timing calibration with the early LHC beam data.

\section{The ATLAS Level-1 Muon Trigger IN The CENTRAL REGION (Barrel)}

\section{A. The ATLAS detector at LHC}

$\mathbf{T}$ HE ATLAS detector is one of the experiments at the CERN LHC proton-proton accelerator in Geneva (Switzerland). It is aimed at analyzing different topologies of

e-mail: Giuseppe.Salamanna@cern.ch. physics events resulting from $p-p$ collisions at a Centreof-Mass Energy of $14 \mathrm{TeV}$. It is therefore composed of different particle detecting technologies following a cylindrical symmetry around the LHC beam axis, for measurement of kinematics and properties of charged and neutral particles in a wide momentum range. A detailed description of the ATLAS detector can be found elsewhere [1].

In particular, for muon trigger and tracking, the ATLAS Muon Spectrometer (MS) is used, which covers a wide pseudorapidity region both for muon trigger and offline reconstruction purposes [2]. Here we refer to muons triggered and reconstructed in the central part of the MS, the Barrel, covering $|\eta| \leq 1.05$. This region is instrumented with a series of Monitored Drift Tubes (MDT) chambers, for a precise determination of the muon kinematics. Attached to the middle and outer layers of the MDT chambers, Resistive Plate Chambers (RPC) are installed to provide a fast response and to trigger on the passage of a muon. The raw detector resolution is $\approx 2$ $n s$ for a single hit.

In this paper we describe use of triggers based on different technologies, in particular one using the MS and another based on the Transition Radiation Tracker (TRT), part of the ATLAS Inner Detector [1].

\section{B. The ATLAS Level-1 trigger system}

$\mathbf{T}$ HE Level-1 Muon Trigger system has been designed following the requirements described in [2]. It is part of the ATLAS Level-1 trigger system, which is the first stage of the event selection (followed by the High Level Trigger, consisting of Level-2 and the Event Filter). Beside the RPC trigger in the Barrel, the complete Level-1 system includes the Calorimeters (L1Calo), the muons system in the end-caps made with Thin Gap Chambers (TGC), the Muon Central Trigger Interface (MuCTPI), the Central Trigger Processor (CTP) and the TTC, as signal processing and distribution system. The Level-1 system has to cope with a $40 \mathrm{MHz}$ bunch crossing rate (dictated by the $25 \mathrm{~ns}$ LHC bunch crossing $(B C)$ frequency), having to reduce it to a rate of $100 \mathrm{kHz}$, acceptable at the following trigger levels.

The commissioning of the complete system, started during 2007 , included different steps of test and optimization which concluded in the integration, sector by sector, of the complete sub-detector into the ATLAS data taking with cosmic rays. Due to the large acceptance of the detector for cosmic rays and the high configurability of the trigger electronics, the RPC 
trigger has been one of the main triggers for commissioning with cosmic rays.

\section{The Muon Barrel trigger system layout}

$\mathbf{T}$ $\mathrm{HE}$ area covered by the RPC detector is $3650 \mathrm{~m}^{2}$ and the front-end electronics consists of approximately 355,000 readout channels. The overall system is segmented in $\mathbf{6 4}$ trigger sectors, grouped following the detector segmentation in the plane transverse to the proton beam axis: four trigger sectors cover one of the 16 geometrical sectors of the RPC detector (made of 8 large and 8 small sectors). The spatial coincidences are organized in projective and overlapping trigger towers, which are the basic units of the system. Each trigger tower covers a $\Delta \eta \times \Delta \phi=0.2 \times 0.2$ portion of space. A trigger tower is actually implemented as one low $P_{T}$ and one high $P_{T}$ trigger box (called PAD), which process signals coming respectively from the middle and the outer detector stations. Trigger and readout data from the low $P_{T}$ PAD are sent to the $P_{T}$ PAD, which are responsible for the final tagging of the muon trigger candidate with information such as the bunch crossing, the pT threshold and the position within the tower. The information on the $P_{T}$ threshold and the position of the muon candidates are sent to the MuCTPI, which resolves the overlap between different segments of the muon system, as the end-caps and the barrel regions. The CTP accepts both the input from Muon and calorimeter triggers and makes the overall decision, generating the final Level-1 Accept (L1A) signal.

The first level decision is fully provided by hardware logic and, in the muon barrel system, is implemented into custom ASICs, called Coincidence Matrices (CM). It is based on the request for a number of coincidences in the RPC layers lying within a geometrical path, called trigger roads. Their width is related, in the bending view, to the expected $P_{T}$ of the muon coming from the interaction point, while in the transverse view (non-bending plane) this window in optimized in order to suppress correlated noise from cavern background. The trigger coincidences in each tower are identified in the two views independently by different CM ASIC processors. More information can be found in [3].

The trigger selection requires also a temporal coincidence within a programmable time window. During time-in commissioning this window is set to $25 \mathrm{~ns}$, but can be decreased by steps of $1 / 8$ of $\mathrm{BC}$, in order to take advantage of the available time resolution. In the rest of this paper we will address the timing-in of all the Level-1 Muon Barrel Trigger elements.

\section{IMPORTANCE AND ISSUES WITH TIMING FOR THE LEVEL-1 MUON BARREL TRIGGER}

A CORRECT synchronicity among all the various elements of the Level-1 Muon Barrel Trigger is essential, due to the way the steering of the read-out of the various ATLAS sub-detectors is concieved. In fact, after a muon is produced at a time $t_{L H C}$ in a primary interaction at the LHC and passes through an RPC chamber, a trigger is issued at a certain time $t_{0}=t_{L H C}+t_{\text {delay }}$. Both times can be conveniently expressed in units of Bunch Crossing (BC) times, equivalent to $25 \mathrm{~ns}$. Consequently, as soon as a Level-1 trigger is released, a L1A signal is sent to all the sub-detectors and all data stored are read-out.

Therefore, if two or more elements of the Level-1 Muon Barrel Trigger are not well timed in with respect to each other and with the LHC clock $\left(t_{\text {delay }} \leq 25 \mathrm{~ns}\right)$, parts or all of the physics event are assigned to a wrong collision, which results in a high risk to bias the final physics analysis. Moreover, this bias is dependent on which part of the RPC system was involved in the triggering. Due to this, it is of the uttermost importance that the Level-1 Muon Barrel Trigger system be well timed-in as soon as possible within the ATLAS detector commissioning phase, before starting to produce data to be analyzed for physics.

As seen in the previous section, the Level-1 Muon Barrel Trigger is made of several independent elements, each of which works separately in triggering the passage of a muon. In particular, the electronics that apply the trigger logics for each of them are subject to different delays, and need to be timed in.

A delay can be present between one trigger element and another in a different position of ATLAS due to the following reasons:

1 the time that the muon takes to travel from one element to another (Time-of-Flight): depending on the kinematics of the muon, this contribution can be as large as 35ns;

2 irreducible signal propagation along an RPC strip: $\leq$ $12 \mathrm{~ns}$ are necessary for the signal to travel from the point where the muon hit the RPC sensitive strips to the edge where it is read-out;

3 the lenght of the cables from the detector Front-End electronics (detector) to the trigger electronics (PADs), where the coincidence logics is applied in order to issue a trigger candidate; and that of the optical fibers that transport the information of the trigger candidate to the CTP.

The adjustement in time of all of the trigger channels in order to compensate for the above jitters requires setting more than $30 \mathrm{k}$ channels at the level of the CM, to values obtained from our local and global timing analysis.

\section{TIME ALIGNMENT STRATEGY WITH COSMIC RAY DATA}

A S stated, a strategy to align in time the various parts of the Level-1 Muon Barrel Trigger needs to be developed already in preparation for the ATLAS detector commissioning with cosmic ray data: we want to assess and validate the procedure and the necessary tools before the LHC beams. This with the aim of having a large portion of the detector already timed-in by the first beams.

For this purpose, up to 200 Million cosmic ray events collected by ATLAS to date can be used, both selected by standalone Level-1 Muon triggers and a combination of triggers. Performing the timing analysis with cosmic rays has some advantages and disadvantages, with respect to standard collision data. The advantages are:

1 often in cosmic ray events only a muon is present, which makes it is easier to obtain the RPC trigger element in 
space that provided a trigger, by making use of offline reconstructed tracks and selections at the trigger level;

2 cosmic ray events have a much lower track multiplicity than collision events $(85-90 \%$ of events have $\leq 2$ reconstructed muon tracks in the ATLAS Muon Spectrometer $(M S))$

On the other side, a few disadvantages can be pointed out:

1 the arrival time spectrum of cosmic rays is uniform within $1 \mathrm{BC}(=25 \mathrm{~ns})$, and can therefore be triggered systematically too early or too late with respect to its own $\mathrm{BC}$, due to the trigger electronics intrinsic time;

2 the topology of cosmic ray events does not provide a detector coverage as wide as collision data, and therefore a smaller part of the RPC detector can be probed.

The strategy is two-fold, including both a local timing at the level of the RPC strips concurring to make one coincidence in one of the two views, and at the level of a Tower, combining more coincidences; and a global timing, at the level of the trigger Sectors and Towers within one sector.

\section{A. Local timing alignment}

$\mathbf{I}$ $\mathrm{N}$ these proceedings we briefly sketch the local timing model and concentrate on the global stage. The local calibration is the first step of the timing of the trigger: its goal is to align signals from different strips in one trigger tower. In fact, these come from two adjacent RPC chambers, which have different electronics modules and therefore different cable lengths. This is a potential source of asynchronous triggers. From the bottom to the top, the approach is to:

- adjust all detector layers in coincidence in either the $\eta$ or $\phi$ view (one $\mathrm{CM}$ );

- put in time all the CM's in one Tower in order to guarantee that an overall trigger coincidence is satisfied if a muon passed in that tower

In both cases only RPC information is used, and the idea is to consider the relative time distribution between the various strips of one CM: the mean of this distribution, obtained from a gaussian fit, is then shifted to zero in steps of $\frac{1}{8}$ of a BC, that is to say $3.125 \mathrm{~ns}$.

The same process is then repeated for all the CM within a tower, shifting the relative time distribution towards zero. This is a local calibration procedure which is run automatically during each run and the calibration shifts applied to the next run.

\section{B. Global timing alignment}

O $\mathrm{N}$ top of the local calibration, it is necessary to account for the time differences occurring globally between Towers of one Sector or different Sectors. In order to do this, a strategy has been developed that uses both an external Trigger reference, completely independent of the RPC in time; and a reference sector of the RPC as zero for any other sector. The approach with an external trigger is applied furing cosmic ray data taking runs that are triggered by both the RPC and an ad-hoc trigger set-up for the ATLAS detector commissioning with cosmic rays. This makes use of the TRT-based FastOR trigger [6]: due to a dedicated configuration for low-jitter and the TRT small dimensions ( $1.5 \mathrm{~m}$ along the beam axis) it results in a reduced time jitter (maximum $1 \mathrm{BC}$ ) and is therefore ideal as a reference. An important disadvantage of using the TRT comes also from its reduced geometrical dimensions, which correspond to a substantially reduced acceptance to cosmic rays coming from the top of ATLAS with a wide spectrum of angles.

It is for this reason that also a complementary approach is followed, with the most illuminated RPC sector fixed as a time reference and any other sector probed with respect to it. In fact, once the reference sector is aligned with the TRT, this become an iterative procedure, with a higher acceptance. Furthermore, if performed independently, this disentangles the results from the intrinsic TRT Fast-OR jitter of a maximum of one BC.

In both cases it is necessary to correlate strongly the triggers present in an event with the geometrical position of the RPC element that issued that trigger. It is at this stage that one can correctly account and correct for the muon time-of-flight from the reference to the probed RPC elemen. In order to achieve a certain association, we rely on two pieces of information:

1 we select only events with a L1A from the TRT Fast-OR and only one further trigger provided by the RPC; or one $\mathrm{L} 1 \mathrm{~A}$ from the chosen reference RPC sector and only one further RPC trigger;

2 require that the candidate RPC detector element considered to match the RPC trigger in the event be matched to an offline track reconstructed in the MS by the Moore algorithm [4], this way reducing the chance to consider fake trigger hits originated by noise in the RPC detector.

The second request doesn't affect our overall acceptance to events, as the tracking has been measured on cosmic rays to have an efficiency well above $90 \%$ [5].

When applying the second condition only events containing either a single track or two tracks in opposite hemispheres are considered. Fig.1 shows that the event selection of the first point above is already very powerful in suppressing fake hits even without track match, but that the second requirement helps in further eliminating fake trigger hits from one of the two geometrical views, that do not make it into a final trigger candidate.

The events from one data taking period surviving the above selections are then considered for the global alignment calibration: out of these events a map of delays of each pair (Sector, Tower) with respect to the chosen reference is built, in units of $B C$. The delays are then to apply timing corrections at the Tower level, per sector (on top of the local calibrations). It has to be stressed that this procedure, applying a coarse per$B C$ correction, is sensitive to the flat time spectrum of cosmic rays (Sec. IV) due to the intrinsic limitation in statistics, and is therefore expected to leave small tails accounting for border effects.

\section{RESULTS WITH COSMIC RAY DATA}




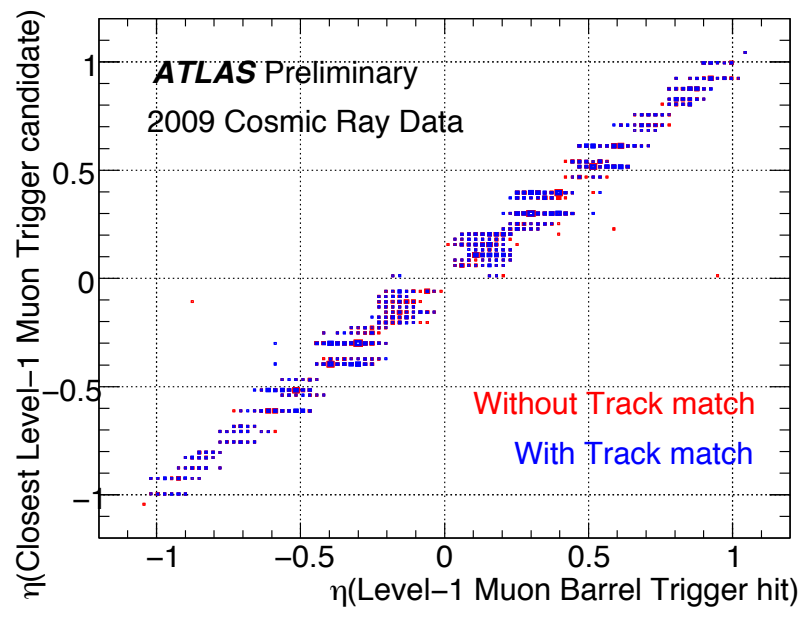

Fig. 1. $\eta$ of the position of the RPC Trigger hit (x-axis) vs the $\eta$ of the position of the closest Level-1 Muon Barrel Trigger candidate, matched by vicinity in $\eta$ and $\phi$ to the CM hit considered. Hits from both $\eta$ and $\phi$ views are plotted here. When the hit is not matched to an offline MS track (red dots), a few hits remain uncorrelated with an actual trigger candidate in the event, being therefore not real triggers (no coincidence satisfied). These disappear when asking for a track match (blue dots).

$\mathbf{O}$ NCE the above procedure is applied, on a pair of test runs from combined and RPC-only runs, we obtain the results in Fig.2, left and right respectively. These are based on a statistics of some hundreds of thousands of selected events. As one can see by comparing the two, the use of an RPC reference is useful to cover sectors around number 60 , which are very poorly probed when the TRT Fast-OR reference is used.

As mentioned, a residual time spread of $1 \mathrm{BC}$ is induced by the intrinsic time spectrum of our data source, while a remainining delay of $>1 \mathrm{BC}$ is the hint that the local calibration didn't fully succeed, due to limited statistics for a given $\mathrm{CM}$ is a Tower.

Finally Fig.3 summarizes the time alignment of the Level-1 Muon Barrel Trigger obtained with respect to the TRT FastOR trigger, for any event (no preliminary selections), after applying the detailed global time calibration, in comparison to the time spread before the calibration. Already approx. $70 \%$ of the trigger elements could be aligned with only one iteration on cosmic-ray data. This can be further refined before collision data arrive, by using a larger statistics from a data-taking run with the very same trigger configuration.

\section{TIMING PLANS WITH LHC BEAMS}

$\mathbf{I}$ $\mathrm{N}$ a few weeks, the first LHC beams will circulate, first in single beam configuration and then with proper collisions. The single beam case will translate, for the detectors, into analyzing events coming from the halo of particles surrounding the actual beam; and the results of the beam being splashed onto a closed collimator, right before the detectors.

In this case, a natural choice of an external reference for our timing studies will be the dedicated ATLAS detection system of the beam passage. This has the advantages that, contrary to cosmic-rays, the beam arrives at an exact time within $25 \mathrm{~ns}$,

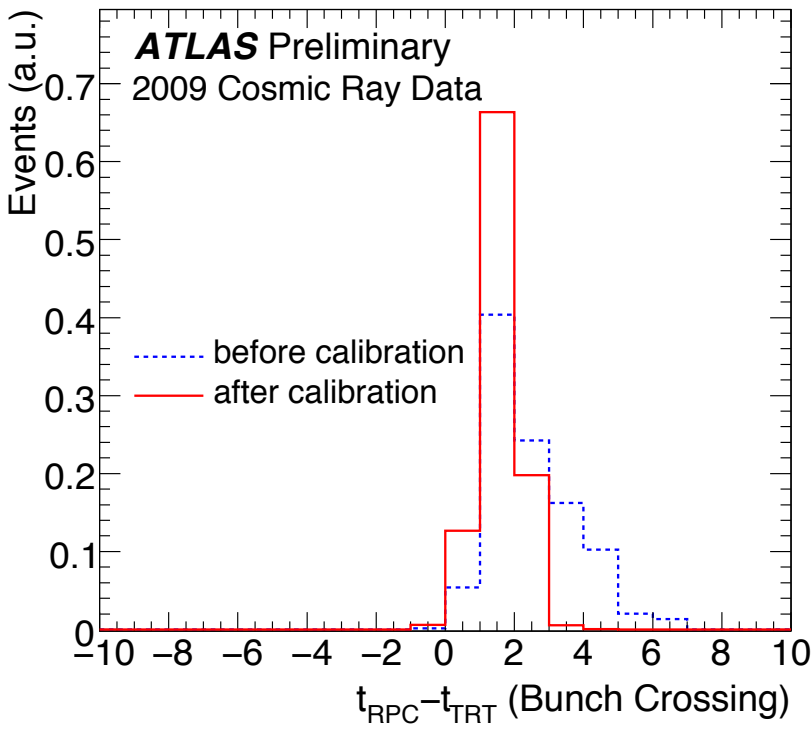

Fig. 3. Bunch-Crossing (BC) distribution of the RPC low-pt trigger, from any trigger sector, with respect to the TRT Fast-OR trigger before and after a single calibration iteration was applied. The delay was set to $1 \mathrm{BC}$ on purpose, to have an RPC trigger right after the TRT L1A.

and is therefore an exact clock. A limitation that will likely prevent our global alignment method from being used as-is for the beam splashes is the very high track multiplicity, that will likely leave us with very few chances to correlate the trigger and RPC detector information to apply corrections.

The plan is to concentrate more on collision data: having exercised and applied our calibration strategy and tools on cosmic data we will be ready to immediately apply them to the first collisions. These can, in fact, are similar the case with the TRT Fast-OR trigger, but with a greatly enhanced statistics.

\section{CONCLUSIONS}

THAVE described here the issue of timing in the ATLAS Level-1 Muon Barrel Trigger system and the vital importance of deploying an effective and well validated time calibration strategy that times in all of the system with respect to the actual LHC Bunch Crossing time altogether. The strategy and tools tested on a large sample of cosmic rays will immediately be applicable to early beam collisions. Results have already been obtained on cosmic rays and $\approx 70 \%$ of the trigger elements could be synchronized even before LHC starts.

\section{ACKNOWLEDGMENT}

The author would like to thank IEEE and the 2009 NSS/MIC Conference organizers for a very professionally organized and interesting conference in a great location. 

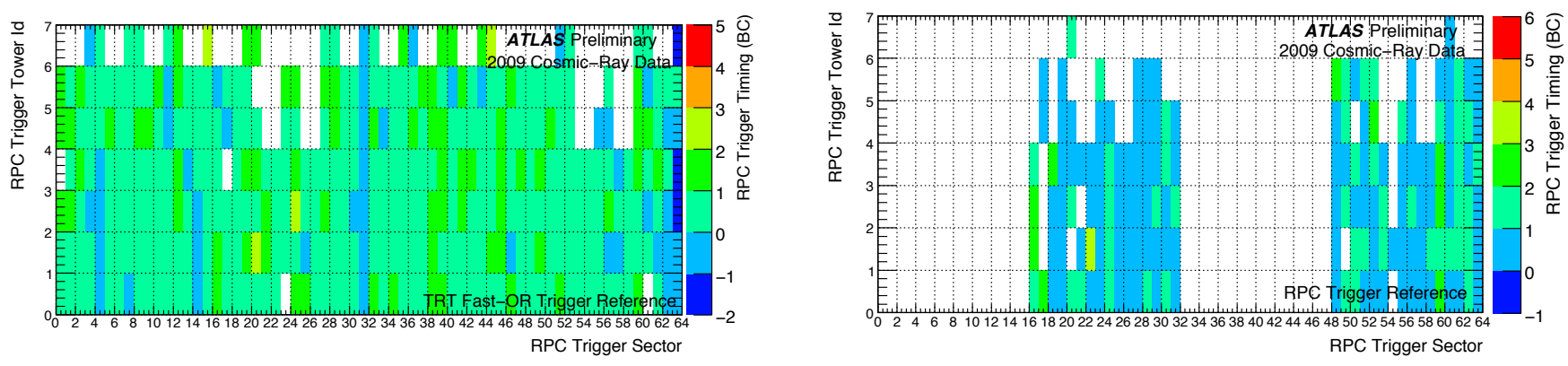

Fig. 2. Left: map (in the Level-1 Muon Barrel Trigger Tower vs Trigger Sector plane) of the delays with respect to the TRT Fast-OR trigger reference, in units of BC. This displays the situation after the recalibration of the Level-1 Muon Barrel Trigger system. Right: Same as Left, but when Sector 39 of the Level-1 Muon Barrel Trigger is used as time reference: only sectors from the lower hemisphere are shown in the plot.

\section{REFERENCES}

[1] The ATLAS Collaboration Expected Performance of the ATLAS Experiment - Detector, Trigger and Physics. arXiv:0901.0512. Available: http://arxiv.org/

[2] The ATLAS Collaboration ATLAS Muon Spectrometer TDR. CERN/LHCC 97-22. 5 June 1997

[3] Anulli, F et al, Study of the Performances of the Level-1 Trigger System for Muons in the Barrel of ATLAS with Cosmic Rays. Proceedings of the 2008 IEEE NSS/MIC Conference.

[4] The ATLAS Collaboration, Expected Performance of the ATLAS Experiment - Detector, Trigger and Performance. CERN-OPEN-2008-20. Available: http://cds.cern.ch/

[5] The ATLAS Collaboration Results from the ATLAS Muon Spectrometer Commisioning with Cosmic Rays. In preparation

[6] Fratina, S et al, The TRT Fast-OR Trigger. Atlas internal note: ATLCOM-INDET-2009-042. A public note is in preparation. 